\title{
Design and Implementation of Capacitive Sensor Readout Circuit on Glass Substrate for Touch Panel Applications
}

\author{
Tzu-Ming Wang ${ }^{1}$ and Ming-Dou Ker ${ }^{1,2}$ \\ ${ }^{1}$ Institute of Electronics, National Chiao-Tung University, Hsinchu, Taiwan. \\ ${ }^{2}$ Department of Electronic Engineering, I-Shou University, Kaohsiung, Taiwan.
}

\begin{abstract}
A capacitive sensor readout circuit on glass substrate for touch panel applications has been designed and fabricated in a 3- $\mu \mathrm{m}$ low temperature poly-silicon (LTPS) technology. In this work, the small voltage difference from capacitance change due to the touch event on panel is amplified by the switch-capacitor (SC) technique. In order to reduce the effect from device characteristic variation in LTPS process, the proposed readout circuit applies the corrected double-sampling (CDS) technique to reduce the offset originated from LTPS process variation. To enhance the resolution of touch panel, a 4-bit analog-to-digital converter is utilized to identify the different touch area conditions.
\end{abstract}

\section{INTRODUCTION}

As the developing of digital life and growing of flat display market, low temperature poly-silicon (LTPS) technology has become an important feature for high image quality display due to the high performance and high resolution. Compared with conventional amorphous silicon (a-Si) thin film transistors (TFTs), some characteristics, such as higher carrier mobility, lower threshold voltage, and higher stability, of low temperature poly silicon (LTPS) TFTs can achieve compact, highly reliable, and high resolution for system integration within a display panel [1], [2].

Fig. 1 shows the trends for system-on-panel (SOP) applications. For normal display panel, TABs are connected to the left and bottom sides of a panel with driver ICs. Integration of driver ICs with LTPS TFTs on panel can achieve COG (chip on glass) but it still requires FPC (flexible printed cable) connection on the bottom for other functional circuits. The most common failure mechanism of TFTLCDs, disconnection of the TABs, is therefore decreased significantly with the save in omitting the usage of ICs and all subcircuits integrated on panel. Besides, the cost of panel becomes lower, as well as the higher yield rate can be also achieved [3]-[5]. Such integration technology contributes to shorten the product lead time because the assemblage of CMOS ICs can be eliminated. Currently, such integration has been proceeding from simple digital circuits to the sophisticated ones, and other functional circuits utilized on panel will be also integrated in the future to achieve SOP applications.

Nowadays, touch panel becomes more and more popular for its simplicity and direct interaction with portable products such as satellite navigation devices, mobile phone, personal digital assistants (PDAs), notebook, and so on. Therefore, integrating touch sensing function into glass substrate has attracted much attention in last few years. Touch panels utilized in electronic consumer products are mainly resistive or capacitive. Resistive touch panel exhibits advantages such as cost-effective, consistent, and durability. On the contrary, disadvantages of resistive-type touch panels include serious glare, low transmittance, and single-touch functionality. Besides, capacitive touch panel can realize multi-touch functionality easily which allows user to operate information instruments more intuitively [6]-[7].

However, compared with the requirement as being a switch, polysilicon TFTs requires good electrical stability as being utilized to integrate circuits or systems on panel. Although using LTPS process can enlarge poly-grain size to improve the device performance, it usually accompanies a random device-to-device variation on LCD panel. The harmful effects of irregular grain boundaries, gateinsulator interface defects, and incomplete ion-doping activation in thin poly-silicon channels result in the variation on electrical characteristics of LTPS TFTs. Polysilicon TFTs suffer serious variation of device behavior, especially in mobility and threshold voltage, which are two important features in the realization of onpanel circuit integration [8], [9].

In this work, a new capacitive sensor readout circuit on glass substrate for touch panel application has been designed and fabricated in a $3-\mu \mathrm{m}$ low temperature poly-silicon (LTPS) technology [10],[11]. By applying the switch-capacitor (SC) circuit, the voltage difference from capacitance change due to the touch events can be amplified. In addition, the corrected double-sampling (CDS) technique is also employed to reduce the offset originated from process variation. To further identify the different touch area during the touch events, a 4-bit analog-to-digital converter (ADC) is used to converter the output of readout circuit into 4-bit digital codes.

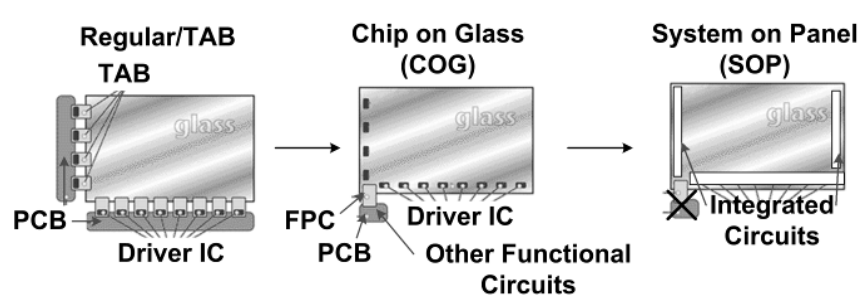

Fig. 1. Trends for system-on-panel (SOP) applications.

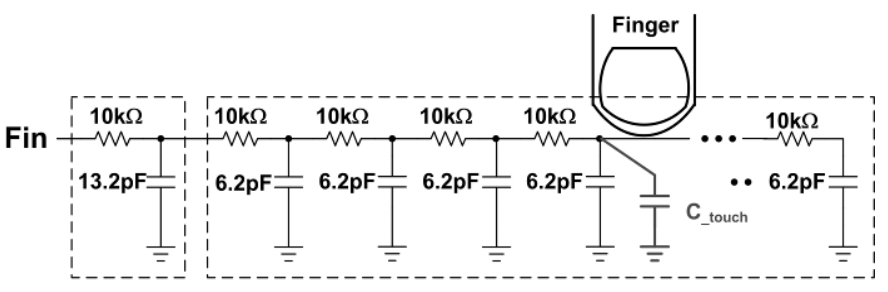

Fanout

14 sensor pads 
Fig. 2. The equivalent RC model of one capacitive sensor line on a 2.8 inch touch panel.

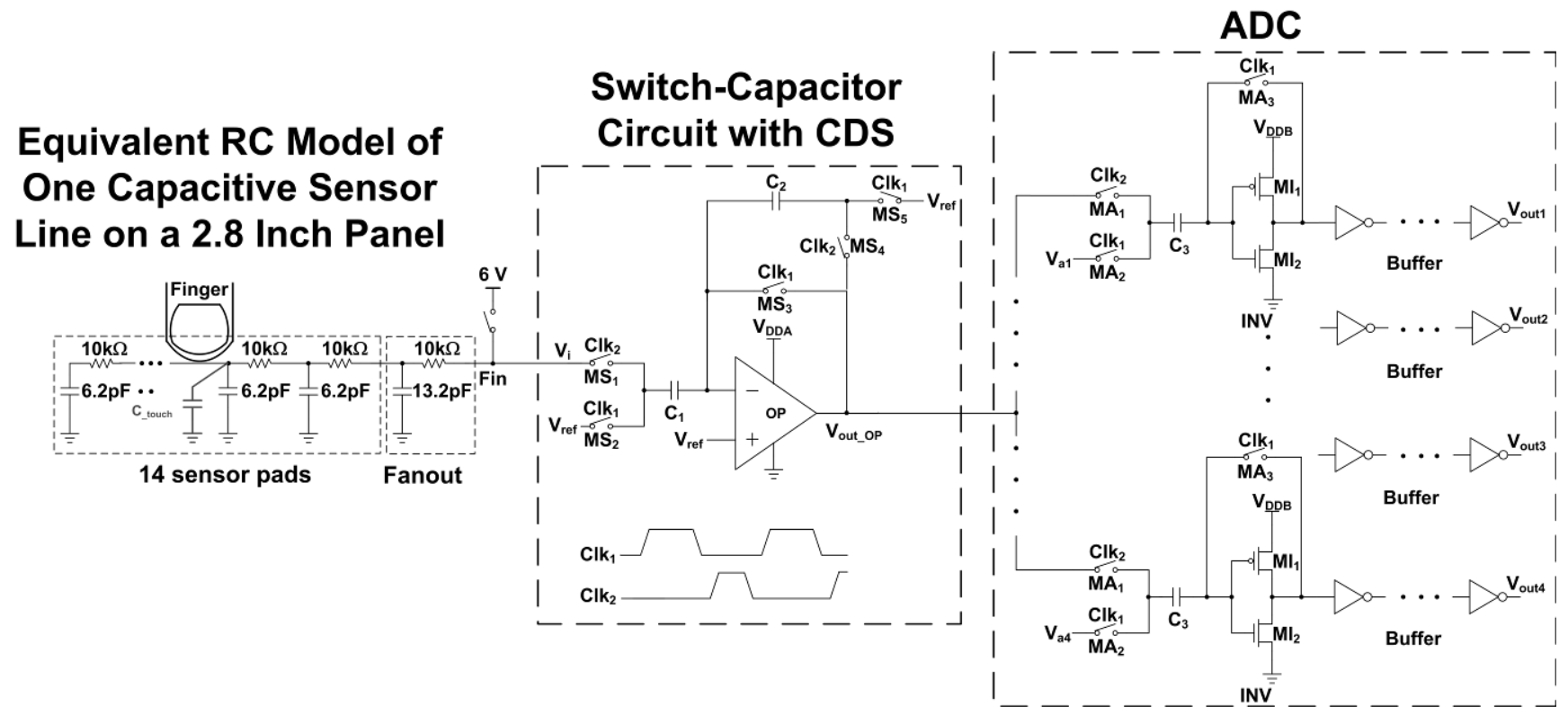

Fig. 3. The new proposed capacitive sensor readout circuit on glass substrate for touch panel applications in a 3- $\mu$ m LTPS technology.

\section{New Proposed On-PANel Readout Circuit}

\section{A. Equivalent Model of the Capacitive Sensor Line}

A capacitive touch panel utilized in LTPS process consists of an insulator glass, coated with a transparent conductor indium tin oxide (ITO). When the conductive objects such as fingers or metal stylus touch the surface or panel, it induces a small capacitance change on the sensor line and can be regarded as a signal to distinguish whether the panel is touched or not. The equivalent RC model of one capacitive sensor line on a 2.8 inch touch panel provided by the panel manufactory with total R of $150 \mathrm{k} \Omega$ and $\mathrm{C}$ of $100 \mathrm{pF}$ is shown in Fig. 2. The Fanout block is the equivalent parasitic RC network of the interconnect line between the sensor line to the output node Fin. The touch capacitor $\left(\mathrm{C}_{\text {touch }}\right)$ is varied from $0.5 \mathrm{pF}$ to $2 \mathrm{pF}$ according to the different touch area. When the sensor line is touched by the finger, $\mathrm{C}_{\text {touch }}$ is added in parallel to the touched node and the total capacitance on the capacitive sensor line is also changed. In order to discriminate between the touch and non-touch events, that is, to detect the capacitance change from $\mathrm{C}_{\text {touch }}$, each node on the sensor line is pre-charged to $6 \mathrm{~V}$ at the beginning. When the touch event happened, the voltage at the output node Fin $\left(\mathrm{V}_{\mathrm{Fin}}\right)$ will be changed to

$$
\mathrm{V}_{\mathrm{FIN}}=\frac{\mathrm{C}_{\text {total }}}{\mathrm{C}_{\text {total }}+\mathrm{C}_{\text {_touch }}} \cdot \mathrm{V}_{\text {pre-charge }} \text {, }
$$

where $\mathrm{V}_{\text {pre-charge }}=6 \mathrm{~V}, \mathrm{C}_{\text {total }}=100 \mathrm{pF}, \mathrm{C}_{\text {touch }}=0.5 \mathrm{pF}-2 \mathrm{pF}$. Therefore, the voltage level at output node Fin under the touch event can be derived from $5.88 \mathrm{~V}$ to $5.97 \mathrm{~V}$ with the corresponding $\mathrm{C}_{\text {touch }}$ value from $2 \mathrm{pF}$ to $0.5 \mathrm{pF}$. For such a capacitive sensor line, the capacitance change due to the touch event can be indicated by the voltage change. So the on-panel readout circuit is designed to distinguish the voltage difference at the Fin node. In this work, the switch-capacitor (SC) technique is applied to enlarge the voltage difference from capacitance change in the touch panel and the corrected double-sampling (CDS) technique is employed to reduce the offset owing to process variation. In addition, the different touch area is further identified by the 4-bit digital output code from onpanel analog-to-digital converter (ADC).

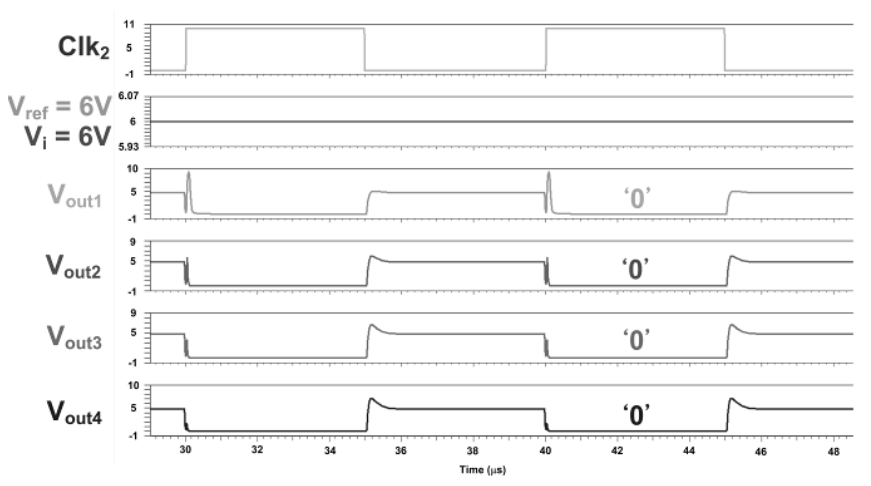

Fig. 4. The simulated result of the proposed circuit under the nontouch event with the digital output code of ' 0000 '.

\section{B. Circuit Implementation and Simulation}

Fig. 3 shows the new proposed on-panel readout circuit for touch panel applications in a 3- $\mu \mathrm{m}$ LTPS technology. The proposed circuit is designed to detect the capacitance change from the capacitive sensor line shown in Fig. 2. The proposed circuit is composed of two parts. One is the switch-capacitor (SC) circuit with corrected double sampling (CDS) technique, and the other is on-panel analog-todigital converter (ADC).

The SC technique is applied to amplify the small voltage difference between $\mathrm{Vi}$ and Vref with the factor of $\mathrm{C} 1 / \mathrm{C} 2$, where $\mathrm{Vi}$ is the voltage from the output node (Fin) of the sensor line. Since the TFTs suffer large device variation in LTPS process compared with that in CMOS silicon process, the CDS technique is utilized to eliminate the offset of operation amplifier (OP). The effect of the input offset can be modeled as an error voltage source (Vos) placed in series with the positive input of OP. For on-panel ADC, the SC technique is applied to cancel the influence of threshold voltage variation of TFT device. All switches are controlled by the clock 
signals CLK1 or CLK2. The circuit operation has two steps, (1) storing the logic threshold voltage Vth,log of INV on capacitor, and

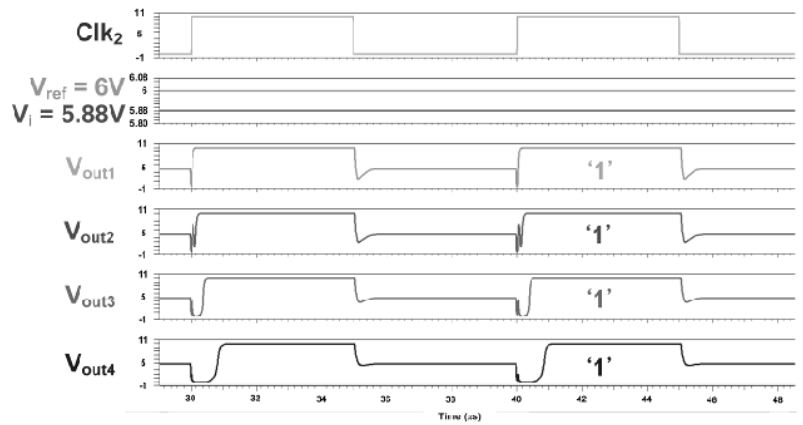

(a)

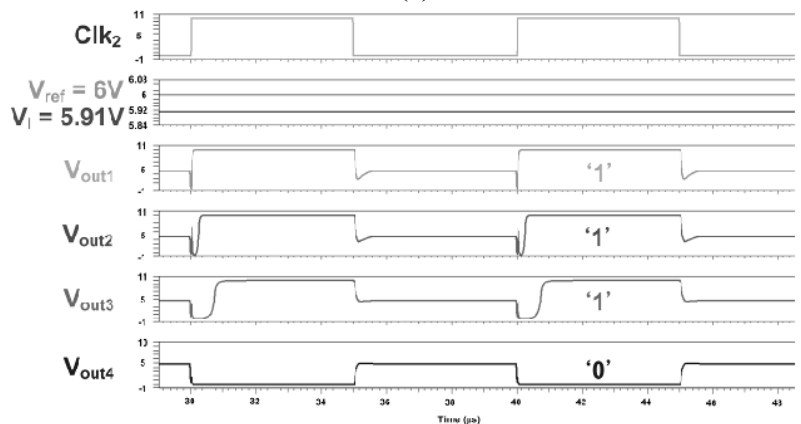

(b)

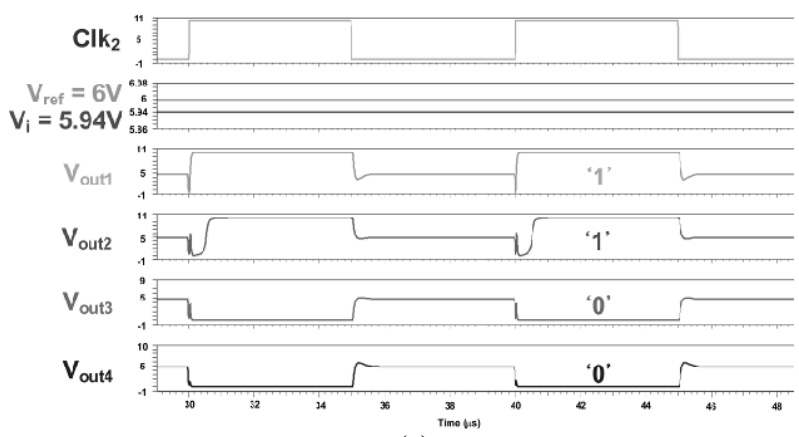

(c)

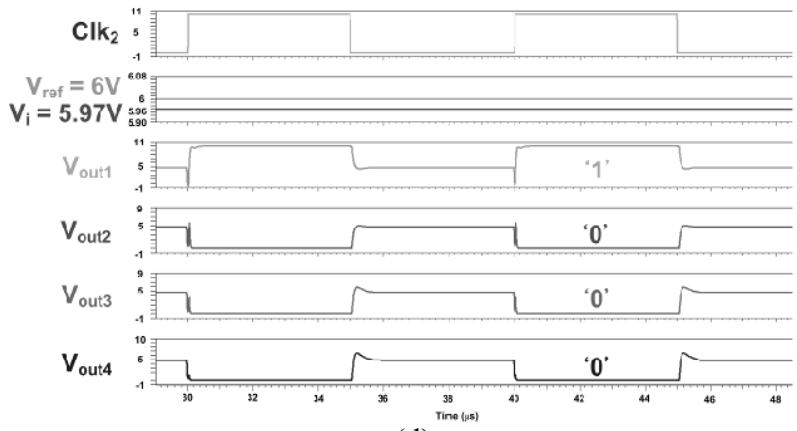

(d)

Fig. 5. The simulated results of the proposed readout circuit with (a) $\mathrm{V}_{\mathrm{i}}=5.88 \mathrm{~V}$ (digital output code: ' 1111 '), (b) $\mathrm{V}_{\mathrm{i}}=5.91 \mathrm{~V}$ (digital output code: '1110'), (c) $\mathrm{V}_{\mathrm{i}}=5.94 \mathrm{~V}$ (digital output code: '1100'), and (d) $V_{i}=5.97 \mathrm{~V}$ (digital output code: ' 1000 '), where the $\mathrm{V}_{\text {ref }}$ is kept at $6 \mathrm{~V}$.

(2) compensating Vth,log and comparing Vout_OP with the reference voltage, Va. In the first step, CLK1 is set to high and the difference between logic threshold voltage Vth,log of inverter and $\mathrm{Va}$ is stored on the capacitor C3. In the second step, CLK1 is switched to low and CLK2 is set to high. Due to charge conservation, the input voltage of inverter becomes $\left(\mathrm{V}_{\text {out } \mathrm{OP}}+\mathrm{V}_{\text {th,log }}-\mathrm{V}_{\mathrm{a}}\right)$. Some buffers are added to guarantee full-swing of the output voltage.

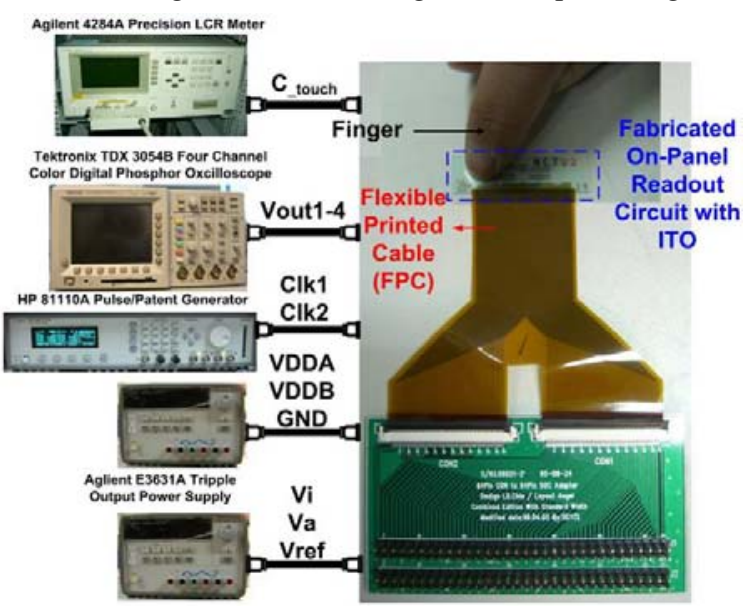

Fig. 6. The fabricated circuits on glass substrate to verify the readout function of the proposed circuit and the corresponding measurement setup

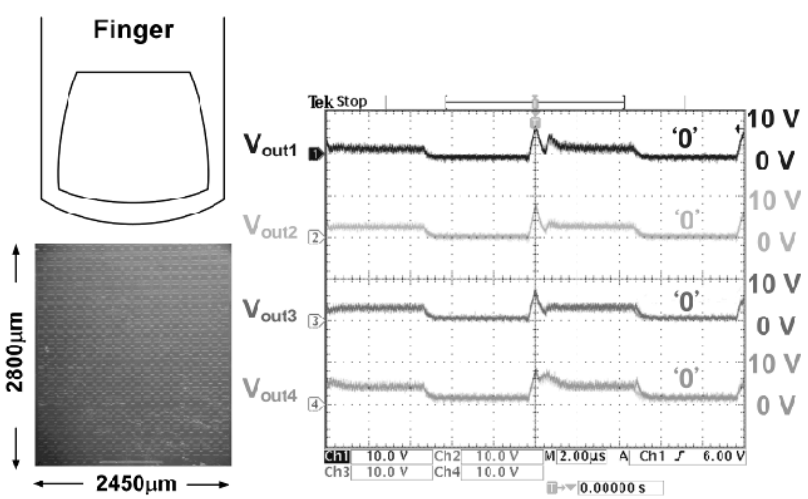

Fig. 7. The measured result of the fabricated circuit under non-touch event.

Fig. 4 shows the simulated result of the proposed circuit under the non-touch event with VDDA $=\mathrm{VDDB}=10 \mathrm{~V}, \mathrm{Vref}=6 \mathrm{~V}, \mathrm{Va1}=$ $6.15 \mathrm{~V}, \mathrm{Va} 2=6.36 \mathrm{~V}, \mathrm{Va} 3=6.6 \mathrm{~V}, \mathrm{Va} 4=6.8 \mathrm{~V}, \mathrm{Clk} 1=\mathrm{Clk} 2=100$ $\mathrm{kHz}, \mathrm{C} 1=8 \mathrm{pF}$, and $\mathrm{C} 2=1 \mathrm{pF}$. By applying the SC and CDS technology, the output voltage of the proposed circuit (Vout1 to Vout4) is only valid when the Clk2 is high. Therefore, the non-touch event of the proposed circuit shows the digital output code of ' 0000 ' when the Clk2 is high. Fig. 5 shows the simulated results under different Vi. The digital code of ADC presents '1111,' '1110,' '1100,' and '1000' under $\mathrm{Vi}=5.88 \mathrm{~V}, 5.91 \mathrm{~V}, 5.94 \mathrm{~V}$, and $5.97 \mathrm{~V}$, respectively.

\section{EXPERIMENTAL RESULTS}

The new proposed circuits have been designed and fabricated in a 3- $\mu \mathrm{m}$ LTPS technology. Fig. 6 shows the fabricated on-panel readout circuit for touch panel application and the corresponding measurement setup. Aglient E3631A tripple output power supply is utilized to supply VDD, Vi, Vref, and Va. HP $81110 \mathrm{~A}$ pulse/patent generator is applied to generate Clk1 and Clk2. Aglient 4284A is utilized to measure $\mathrm{C}$ touch when the fabricated circuit is touch by 
the finger. Fig. 7 shows the measured result of the fabricated circuit under non-touch event, where the digital output code is '0000.' Fig. 8 shows the measured results of the fabricated circuit under different

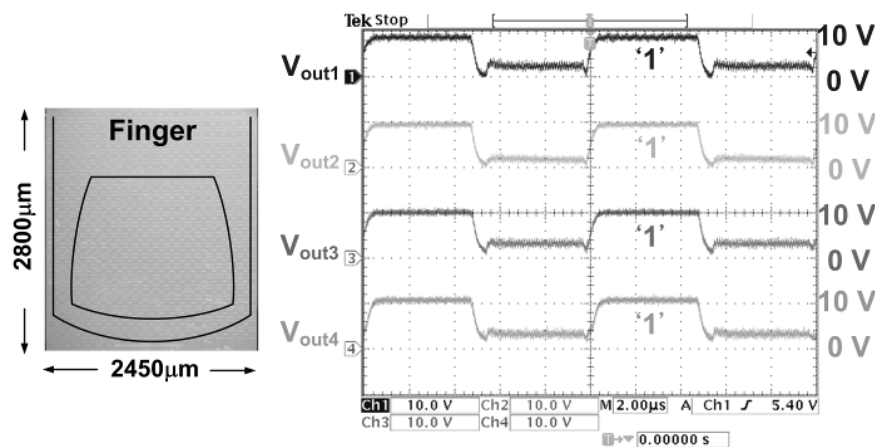

(a)

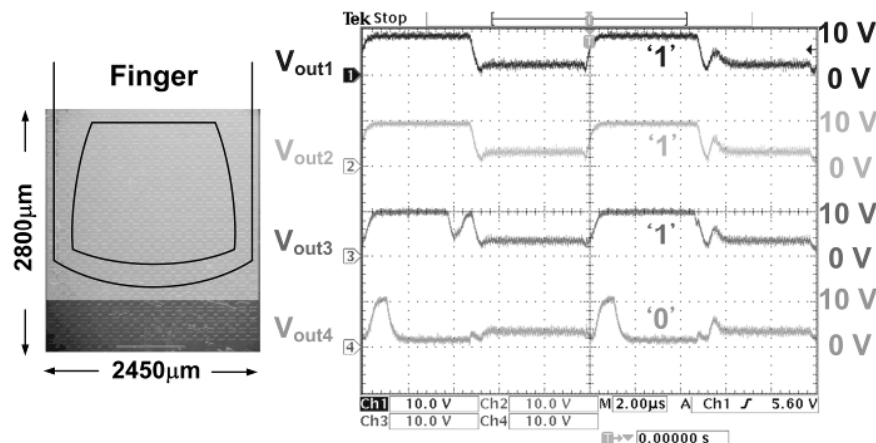

(b)
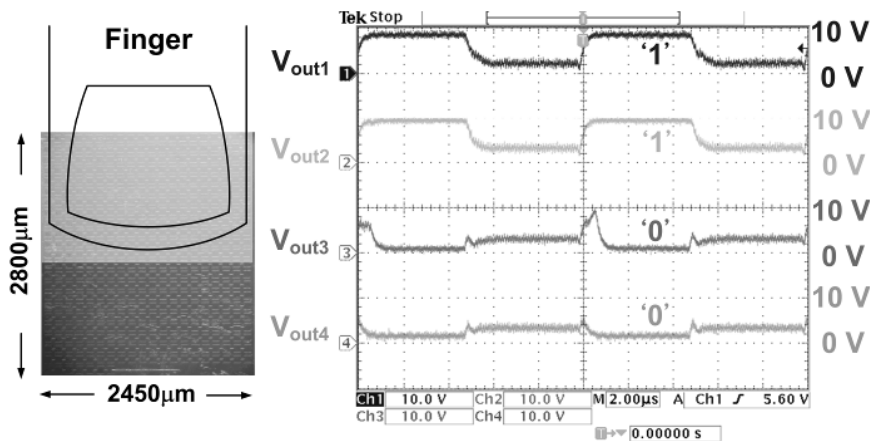

(c)
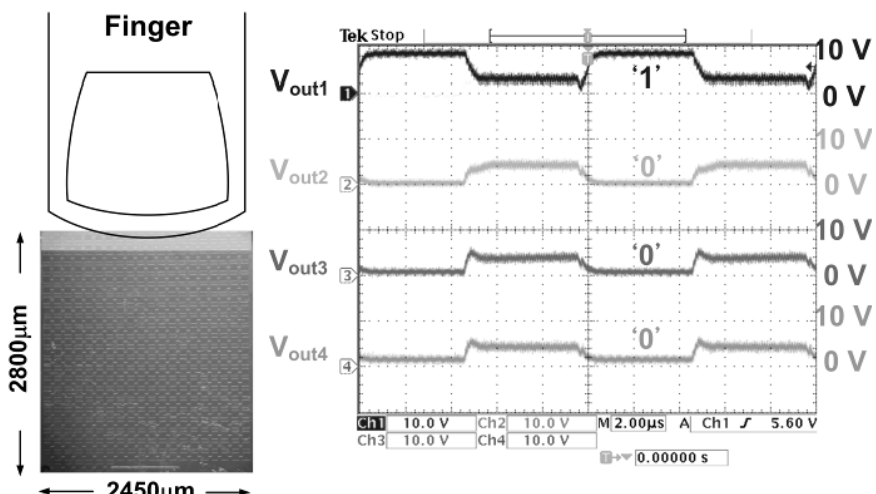

(d)

Fig. 8. The measured results of the fabricated readout circuit under the touched area by finger covered with (a) full, (b) $3 / 4$, (c) $1 / 2$, and (d) less than $1 / 4$ of the ITO area. touch area. The digital output code shows '1111,' '1110,' '1100,' and '1000' when the touched area by finger is covered with full, $3 / 4$, $1 / 2$, and less than $1 / 4$ of the ITO area, respectively.

\section{CONCLUSION}

A new on-panel readout circuit for touch panel applications has been designed and fabricated in a 3- $\mu \mathrm{m}$ low temperature poly-silicon (LTPS) technology. The switch-capacitor (SC) technique is applied to enlarge the voltage difference from the capacitance change of touch panel, and the corrected double-sampling (CDS) technique is also employed to reduce the offset owing to process variation. The different touch area can be identified by the 4-bit digital output. The proposed readout circuit for touch panel application on glass substrate can be integrated in the active matrix LCD (AMLCD) panels to increase the sensing resolution of touch panel for SOP applications.

\section{ACKNOWLEDGEMENT}

This work was supported by AU Optronics Corporation; partially supported by the "Aim for the Top University Plan" of National Chiao-Tung University and Ministry of Education Taiwan, R.O.C.; and partially supported by National Science Council (NSC), Taiwan, under Contract of NSC 98-2221-E-009-113-MY2.

\section{REFERENCES}

[1] K.-H. Lim, H.-W. Kim, J.-S. Kang, Y.-C. Sung, and O.-K. Kwon "A novel 2-stage analog amplifier with self-compensated current loads using low temperature poly-si TFTs for flat panel displays," SID Dig. Tech., 2008, pp. 541-544.

[2] J.-S. Yoon, J.-S. Kang, and O.-K. Kwon "High efficient p-type only cross-coupled DC-DC converter using low temperature poly-si TFTs for mobile display applications," SID Dig. Tech., 2008, pp. 545-548.

[3] AU Optronics Corporation (2010), TFT-LCD Introduction, [Online]. Available: http://www.urt.com.tw/us/rd tft.htm.

[4] C.-S. Tan, W.-T. Sun, S.-H. Lu, C.-H. Kuo, S.-H. Yeh, I.-T. Chang, C.-C. Chen, J. Lee, and C.-S. Yang, "A fully integrated poly-Si TFT-LCD adopting a novel 6-Bit source driver and a novel DC-DC converter circuit," SID Dig. Tech., 2004, pp. 1456-1459.

[5] T. Nishibe and H. Nakamura, "Value-added circuit and function integration for SOG (System-on-Glass) based on LTPS technology," SID Dig. Tech., 2006, pp. 1091-1094.

[6] C.-H. Li, M.-J. Jou and Y.-J. Hsieh, "Multi-touch panel: trend and applications," Proc. IDW, 2009, pp. 2127-2130.

[7] E. Kanda, T. Eguchi, Y. Hiyoshi, T. Chino, Y. Tsuchiya, T. Iwashita, T. Ozawa, T. Miyazawa and T.Matsumoto, "Integrated active matrix capacitive sensors for touch panel LTPS-TFT LCDs," SID Dig. Tech., 2008, pp. 834-837.

[8] W.-Y. Guo, C.-Y. Meng, A. Shih, and Y.-M. Tsai, "Reliability of low temperature poly-Si thin film transistor," in IDMC Tech. Dig., 2003.

[9] M.-D. Ker, C.-K. Deng, and J.-L. Huang, "On-panel output buffer with offset compensation technique for data driver in LTPS technology," IEEE J. Display Tech., vol. 2, no. 2, pp. 153-159, Jun. 2006.

[10] T.-M. Wang, M.-D. Ker, Y.-H. Li, C.-H. Kuo, C.-H. Li, Y.-J. Hsieh, and C.-T. Liu, "Design of on-panel readout circuit for touch panel application," SID Dig. Tech., 2010, pp. 1933-1936.

[11] T.-M. Wang and M.-D. Ker, "Design and implementation of readout circuit on glass substrate for touch panel applications," IEEE/OSA Journal of Display Technology, vol. 6, no. 8, pp.290297, Aug. 2010. 